Proc. of the X Int. Conf. - Ion Implantation and other Applications of Ions and Electrons, Kazimierz Dolny 2014

\title{
Ion-Induced Modification of Structure and Magnetic Anisotropy in Granular FeCoZr- $\mathrm{CaF}_{2}$ Nanocomposite Films
}

\author{
J. Kasiuk ${ }^{a}$, J. Fedotova ${ }^{a}$, J. Przewoznik ${ }^{b}, \mathrm{Cz}_{\text {. Kapusta }}{ }^{b}, \mathrm{~V} . \mathrm{Skuratov}^{c}$, \\ M. Milosavluevic ${ }^{d}$, V. Bondariev ${ }^{e}$ And T.N. Koltunowicz ${ }^{e, *}$ \\ ${ }^{a}$ NC PHEP Belarusian State University, 220088 Minsk, Belarus \\ ${ }^{b}$ AGH University of Science and Technology, 30-059 Kraków, Poland \\ ${ }^{c}$ Joint Institute for Nuclear Research, 141980 Dubna, Russia \\ ${ }^{d}$ VINČA Institute of Nuclear Sciences, P.O. Box 522, 11001 Belgrade, Serbia \\ ${ }^{e}$ Lublin University of Technology, 20-618 Lublin, Poland
}

\begin{abstract}
The paper reports on the results of structural analysis and magnetometry of granular nanocomposite films $\mathrm{FeCoZr}-\mathrm{CaF}_{2}$ irradiated with $\mathrm{Xe}$ and $\mathrm{Kr}$ ions at different fluences. The observed effect of enhanced perpendicular magnetic anisotropy characterizing pristine films is discussed with respect to the irradiation regimes and structural changes of the films originating from the impact of ions.
\end{abstract}

DOI: 10.12693 /APhysPolA.128.828

PACS: 75.50.Tt, 75.30.Gw, 81.15.Jj, 81.40.Wx, 81.40.Rs, 61.80.Jh

\section{Introduction}

Nanostructured films exhibiting perpendicular magnetic anisotropy are promising materials for high density recording media, magnetic field sensors, and systems for visualization of magnetic field distribution [1]. It was previously reported that oriented nanostructures could be successfully grown in the films by ion-beam sputtering techniques at specific deposition regimes $[2,3]$. Optimal choice of experimental conditions as well as chemical composition of nanoparticles and matrix allows fabrication of elongated, columnar-like magnetic structures oriented nearly perpendicular to the film surface and exhibiting perpendicular magnetic anisotropy. However, imperfection of this method for synthesis of oriented nanostructures is related to some deviations of particles long-axes orientation from normal to the film plane (angular directional dispersion) as well as to the uneven size of "nanocolumns" and their distribution inside matrix. This work is focused on the irradiation of composite films with high-energy heavy ions in order to promote perpendicular magnetic anisotropy by homogenization of elongated nanoparticles orientation.

\section{Experimental}

Granular $\left(\mathrm{Fe}_{45} \mathrm{Co}_{45} \mathrm{Zr}_{10}\right)_{x}\left(\mathrm{CaF}_{2}\right)_{100-x} \quad(x=58$ 73 at.\%) nanocomposite films synthesized by ion-beam sputtering in $\mathrm{Ar}$ atmosphere on $\mathrm{Al}$ foil and demonstrating perpendicular magnetic anisotropy [3] are irradiated by $167 \mathrm{MeV} \mathrm{Xe}^{26+}$ and $107 \mathrm{MeV} \mathrm{Kr}{ }^{17+}$ ions with fluence $D$ in the range $7 \times 10^{12}-10^{14}$ ion $/ \mathrm{cm}^{2}$ generated

*corresponding author; e-mail: t.koltunowicz@pollub.pl by IC-100 heavy ion cyclic accelerator (JINR, Dubna). Orientation of ion beam is along the normal to the films plane.

X-ray diffraction (XRD) analysis is performed by an Empyrean PANalytical diffractometer using a diffracted beam graphite monochromator and an X'Celerator linear detector $\left(\mathrm{Cu} K_{\alpha}\right.$ radiation). The data are collected with divergent-beam optics (using 1/32" divergence and 1/16" anti-scatter slits) at a grazing incidence of 5 degrees with respect to the sample surface, with the detector scanning the $2 \theta$ space over the $10-120$ degree range.

The dc magnetic moments $(\mu)$ of films are studied using the vibrating sample magnetometer (VSM) option of a Quantum Design PPMS in the range of magnetic fields $H=0-90 \mathrm{kOe}$ and temperature $T=2-300 \mathrm{~K}$. During measurements magnetic field is applied in the direction parallel $\left(H_{\|}\right)$or perpendicular $\left(H_{\perp}\right)$ to the films surface.

\section{Results and discussion}

The XRD patterns of $(\mathrm{FeCoZr})_{x}\left(\mathrm{CaF}_{2}\right)_{100-x}$ films with $x=58$ and 73 irradiated with $D=7 \times$ $10^{12}-10^{13}$ ion $/ \mathrm{cm}^{2}$ as well as the XRD patterns of pristine films are presented in Fig. 1. Pristine films contain two phases including dominating contribution of bcc $\alpha$ $\mathrm{FeCo}(\mathrm{Zr})$ crystalline nanoparticles and traces of $\mathrm{CaF}_{2}$ matrix [3] disappearing at $x=73$ because of its low content or amorphous state. Figure 1a reveals that irradiation of $x=58$ film with $D=7 \times 10^{12} \mathrm{ion} / \mathrm{cm}^{2}$ does not destroy crystalline structure of $\alpha$ - $\mathrm{FeCo}(\mathrm{Zr})$ nanoparticles. It even leads to narrowing of peaks corresponding to this phase and increase of their intensities. Similarly, no essential changes in structure and phase composition of $x=73$ film are detected after irradiation with $D=8 \times 10^{12}$ ion $/ \mathrm{cm}^{2}$ (Fig. 1b). 


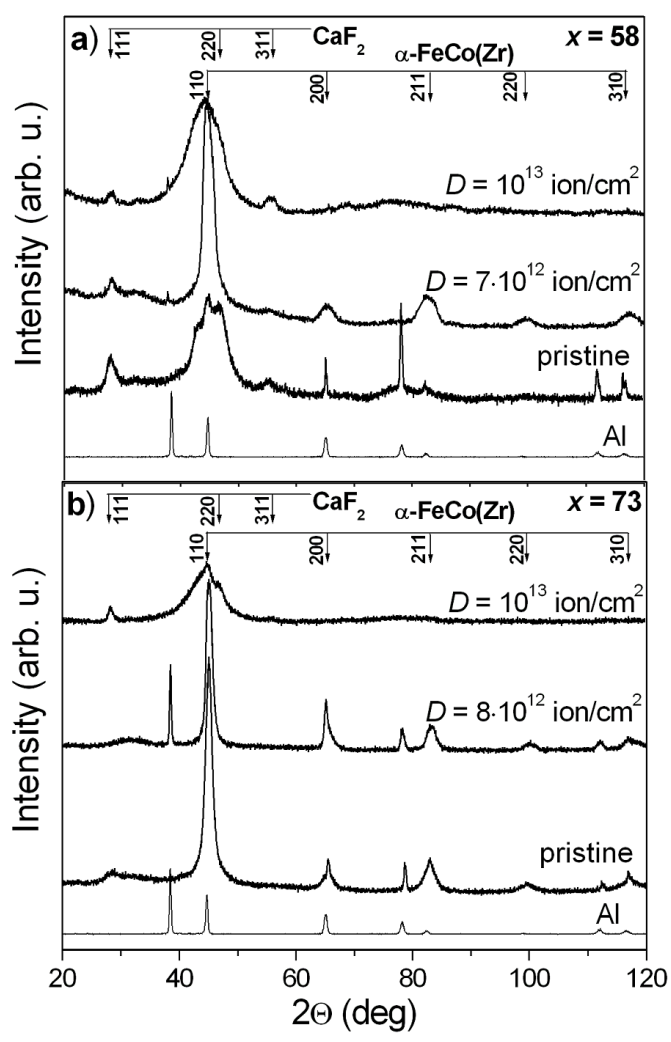

Fig. 1. XRD patterns of $(\mathrm{FeCoZr})_{x}\left(\mathrm{CaF}_{2}\right)_{100-x}$ films with $x=58$ (a) and $x=73(\mathrm{~b})$, pristine and after irradiation with $167 \mathrm{MeV}$ Xe ions of fluence $D=7 \times$ $10^{12}-1 \times 10^{13}$ ion $/ \mathrm{cm}^{2}$.

The XRD patterns of both $x=58$ and 73 films irradiated with the higher fluence $D=1 \times 10^{13}$ ion $/ \mathrm{cm}^{2}$ shows significant changes in their structure compared to the pristine samples (Fig. 1). The evident broadening of peaks corresponding to $\alpha-\mathrm{FeCo}(\mathrm{Zr})$ phase and almost complete disappearance of (200), (211), (220) and (310) reflections indicate strong disordering of nanoparticles structure. In contrast to that, reflections from $\mathrm{CaF}_{2}$ phase became more evident after irradiation, especially in the case of the $x=73$ film.

A possible reason for such a significant difference in structure of the films irradiated under two regimes $(D=$ $7-8 \times 10^{12}$ and $\left.1 \times 10^{13} \mathrm{ion} / \mathrm{cm}^{2}\right)$ is that at higher fluence overlapping of ion track regions takes place. Ion tracks are the regions in the film with high structural disordering induced by ions impact. Simple calculations for tracks with $1.5 \mathrm{~nm}$ in diameter give the value of critical fluence $D_{\mathrm{C}} \approx 10^{13}$ ion $/ \mathrm{cm}^{2}$ separating the regimes of isolated single-tracks and overlapping ion tracks formation.

The XRD analysis of $x=73$ film irradiated with $107 \mathrm{MeV} \mathrm{Kr}{ }^{+}$ions of $D=10^{14}$ ion $/ \mathrm{cm}^{2}$ (not shown) is in a good agreement with that one for $x=73$ film irradiated with $167 \mathrm{MeV} \mathrm{Xe}^{+}$ions of $D=10^{13}$ ion $/ \mathrm{cm}^{2}$ (Fig. 1b). This means that such a fluence is above-critical despite lower energy of ions. However, no complete destruction (or amorphization) of $\alpha-\mathrm{FeCo}(\mathrm{Zr}$ ) nanoparticles structure is observed even under irradiation with such a high fluence.

Modification of magnetic properties of $(\mathrm{FeCoZr})_{73}\left(\mathrm{CaF}_{2}\right)_{27}$ film is analyzed for different regimes (fluences) of irradiation. Field dependences of magnetic moment $\mu$ measured at $H_{\|}$for the studied films irradiated by $\mathrm{Xe}^{+}$ions with two fluences $D=8 \times 10^{12} \mathrm{ion} / \mathrm{cm}^{2}$ (below $D_{\mathrm{C}}$ ) and $D=1 \times 10^{13} \mathrm{ion} / \mathrm{cm}^{2}$ (above $D_{\mathrm{C}}$ ) together with $\mu(H)$ dependences for pristine $x=73$ film are presented in Fig. 2a. Magnetometry, the Mössbauer spectroscopy and microstructure analysis reported previously in $[3,4]$ indicate that the pristine film exhibits perpendicular magnetic anisotropy originating from the shape of nanoparticles and their alignment along to the direction of film normal (nanocolumns). This is evidenced, among others, by high anisotropy field $H_{\mathrm{a}}$ in the film plane of $\approx 1.5 \mathrm{kOe}$ (Fig. 2a). $H_{\mathrm{a}}$ is the field which is necessary to magnetize sample in the hard direction (at $90^{\circ}$ to the easy axis of elongated nanoparticles) [5]. It is worth noting that for isotropic granular metaldielectric films with high $x$ value, saturation field is determined generally by the magnetic properties of bulk material of nanoparticles. For $\alpha$ - $\mathrm{FeCo}(\mathrm{Zr})$ it corresponds to $\approx 0.3-0.6 \mathrm{kOe}[6]$.

As can be seen in Fig. 2a, irradiation with lower (under-critical) fluence $D=8 \times 10^{12} \mathrm{ion} / \mathrm{cm}^{2}$ leads to the essential increase of $H_{\mathrm{a}}$ value which exceeds $2 \mathrm{kOe}$ at $2 \mathrm{~K}$ as compared to $H_{\mathrm{a}} \approx 1.5 \mathrm{kOe}$ for the initial film. The shape of $\mu(H)$ curves also changes. Namely, after irradiation, magnetization curves consist of two almost linear regions joining at $H_{\mathrm{a}}$. Low field linear increase of $\mu$ up to $H_{\mathrm{a}}$ evidences that such orientation of magnetic field (at $H_{\|}$) corresponds to the hard magnetization direction. The increase of $H_{\mathrm{a}}$ after irradiation can, therefore, be attributed to the enhancement of perpendicular magnetic anisotropy in the film. It is associated with ion-induced refinement of metallic nanocolumns arrangement inside matrix perpendicular to the surface when single ion tracks "cut" nanoparticles that deviate from normal orientation.

In contrast to that, irradiation of the $x=73$ film with higher (above-critical) fluence $D=1 \times 10^{13} \mathrm{ion} / \mathrm{cm}^{2}$ does not lead to such significant changes in the shape of magnetization curves (Fig. 2a). However, $H_{\mathrm{a}}$ also increases after irradiation and its value is comparable with that obtained after irradiation with $D=8 \times 10^{12}$ ion $/ \mathrm{cm}^{2}$, especially in the case of low temperature $\mu(H)$ curves $(T=2-100 \mathrm{~K})$.

The $\mu(H)$ dependences of irradiated $x=73$ film measured at $H_{\perp}$ are presented in Fig. 2b for the same irradiation conditions. The main peculiarity of $\mu(H)$ curves obtained after irradiation with $D=8 \times 10^{12} \mathrm{ion} / \mathrm{cm}^{2}$ as compared to the pristine film is the decrease of demagnetizing field $H_{\mathrm{d}}$ from $12 \mathrm{kOe}$ down to $9 \mathrm{kOe}$. High $H_{\mathrm{d}}$ parameter is usually observed in the normal direction to the film plane for the films with high value of magnetization. The highest $H_{\mathrm{d}}$ value corresponds then to uniform 
ferromagnetic film. Thus, a decrease of $H_{\mathrm{d}}$ after irradiation indicates decrease of planar contribution to the magnetic anisotropy due to the shape of film with high volume fraction of ferromagnetic material (about 0.59). A possible reason for that is partial destruction of ferromagnetic nanoparticles by single ion tracks that causes
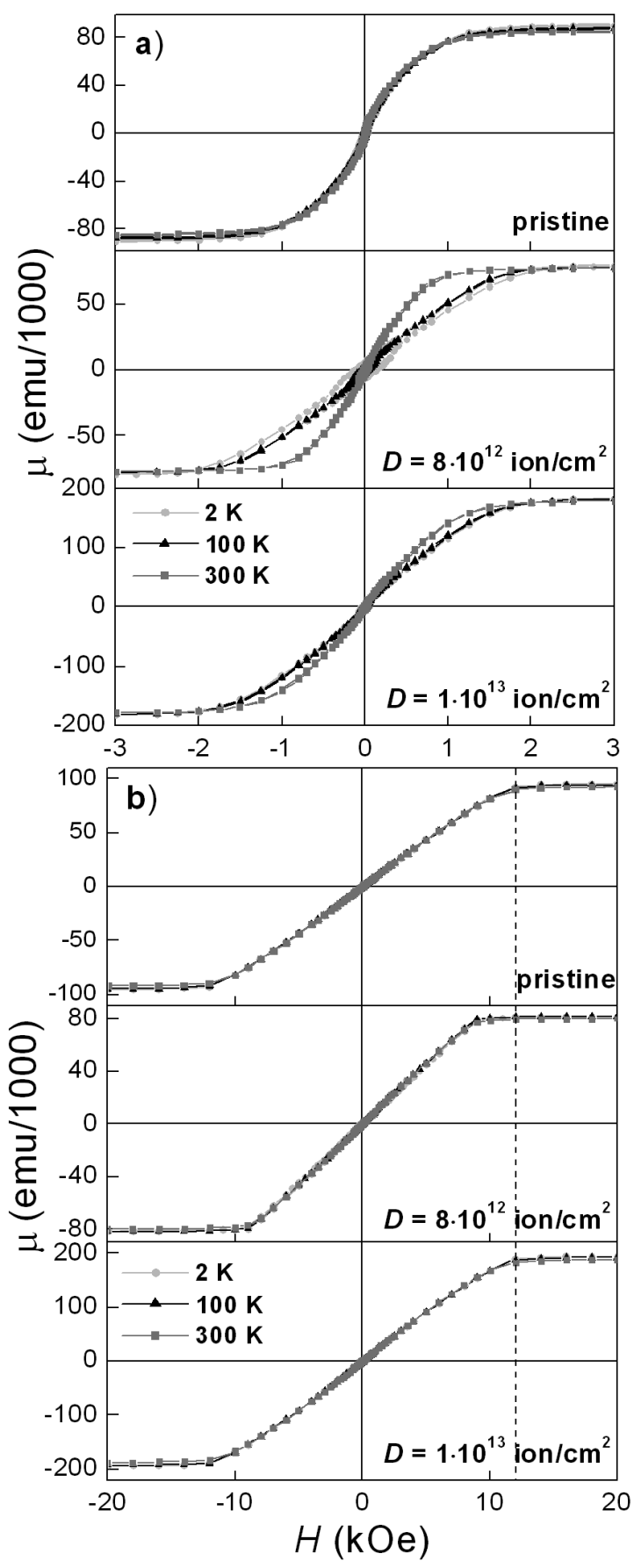

Fig. 2. Parts of field dependencies of magnetic moment $\mu(H)$ at various temperatures for $(\mathrm{FeCoZr})_{7}\left(\mathrm{CaF}_{2}\right)_{27}$ films, pristine and irradiated with $167 \mathrm{MeV}$ Xe ions of $D=8 \times 10^{12}$ and $1 \times 10^{13} \mathrm{ion} / \mathrm{cm}^{2}$, measured in the field parallel (a) and perpendicular (b) to films planes. Dashed line in (b) is guide for eyes corresponding to $H_{\mathrm{d}}$ of the pristine film. formation of additional non-magnetic gaps between elongated nanoparticles.

Additionally, coercive field $H_{\mathrm{C}}$ of the film studied increases considerably from 56 to 116 Oe at $H_{\perp}$ after irradiation with $D=8 \times 10^{12}$ ion $/ \mathrm{cm}^{2}$. This also confirms the enhancement of perpendicular magnetic anisotropy [5] resulting from ions impact.

Irradiation of $x=73$ films with $D=10^{13}$ ion $/ \mathrm{cm}^{2}$ does not cause any change of $H_{\mathrm{d}}$ but leads to a decrease of $H_{\mathrm{C}}$ in the normal direction to the film $\left(H_{\perp}\right)$ from 56 Oe for pristine film to 39 Oe (Fig. 2b). The latter may indicate some amorphization of ferromagnetic material that is in agreement with the XRD results.

\section{Conclusion}

A systematic analysis of structure, phase composition and magnetic properties of pristine granular $(\mathrm{FeCoZr})_{x}\left(\mathrm{CaF}_{2}\right)_{100-x}$ films $(x=58,73)$ and after their irradiation by $\mathrm{Xe}$ and $\mathrm{Kr}$ ions under different regimes has been carried out. It reveals crucial importance of irradiation fluence $D$ value on the enhancement of the films intrinsic perpendicular magnetic anisotropy induced by nanoparticles shapes as well as on nanoparticles crystalline structure disordering and rearrangement of columns structure. A critical value $D_{\mathrm{C}} \approx 10^{13} \mathrm{ion} / \mathrm{cm}^{2}$ is determined, which separates the regimes when single ion tracks and overlapping ion tracks are formed. After films irradiation with $D=8 \times 10^{12}$ ion $/ \mathrm{cm}^{2}$, that is below $D_{\mathrm{C}}$, essential increase of anisotropy field (from $\approx 1,5$ to $2 \mathrm{kOe}$ ) as well as coercive field in the direction of film normal (from 56 to $116 \mathrm{Oe}$ ) are observed. This is the evidence of perpendicular magnetic anisotropy increase caused by the alignment of magnetic nanocolumns by single ion tracks. The crystalline structure of $\alpha-\mathrm{FeCo}(\mathrm{Zr})$ nanoparticles does not undergo any significant deterioration after irradiation under such conditions.

In contrast to that, irradiation of films with $D=$ $10^{13}-10^{14} \mathrm{ion} / \mathrm{cm}^{2}$, that is above $D_{\mathrm{C}}$, provides essential $\alpha$-FeCo(Zr) structure disordering which is clearly reflected in the broadening and disappearance of some peaks on the XRD patterns. However, irradiation with the above-critical fluence has not such a destructive influence on magnetic anisotropy of pristine films revealing magnetic ordering, which practically does not change after irradiation.

\section{Acknowledgments}

This research was partially funded by:

- the statute tasks of the Lublin University of Technology, Faculty of Electrical Engineering and Computer Science, S-28/E/2015, entitled "Preparation of nanolayers and nanocomposites metal or semiconductor in the dielectric matrix and the study of their electrical and magnetic properties",

- the statute grant for PhD students at the Faculty of Electrical Engineering and Computer Science, 
- the research project No. IP 2012026572 within the Iuventus Plus program of the Polish Ministry of Science and Higher Education in the years 20132015 .

\section{References}

[1] S. Sun, C.B. Murray, D. Weller, L. Folks, A. Moser, Science 287, 1989 (2000).

[2] A.A. Timopheev, I. Bdikin, A.F. Lozenko, O.V. Stognei, A.V. Sitnikov, A.V. Los, N.A. Sobolev, J. Appl. Phys. 111, 123915 (2012).
[3] J.V. Kasiuk, J.A. Fedotova, T.N. Koltunowicz, P. Zukowski, A.M. Saad, J. Przewoznik, Cz. Kapusta, J. Zukrowski, I.A. Svito, J. Alloys Comp. 586, S432 (2014).

[4] J.V. Kasiuk, J.A. Fedotova, J. Przewoznik, J. Zukrowski, M. Sikora, Cz. Kapusta, A. Grce, M. Milosavljevic, J. Appl. Phys. 116, 044301 (2014).

[5] B.D. Cullity, C.D. Graham, Introduction to magnetic materials, Wiley-IEEE Press, New Jersey 2008.

[6] J. Fedotova, J. Kasiuk, J. Przewoznik, Cz. Kapusta, I. Svito, Yu. Kalinin, A. Sitnikov, J. Alloys Comp. 509, 9869 (2011). 